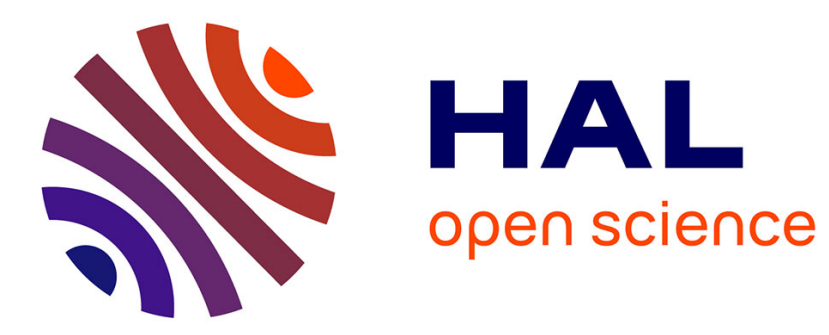

\title{
Asymptotic detection performance of the robust ANMF
} Frédéric Pascal, Jean-Philippe Ovarlez

\section{To cite this version:}

Frédéric Pascal, Jean-Philippe Ovarlez. Asymptotic detection performance of the robust ANMF. 23rd European Signal Processing Conference (EUSIPCO 2015), Aug 2015, Nice, France. pp.524 - 528, 10.1109/EUSIPCO.2015.7362438 . hal-01521934

\section{HAL Id: hal-01521934 \\ https://hal.science/hal-01521934}

Submitted on 27 Mar 2020

HAL is a multi-disciplinary open access archive for the deposit and dissemination of scientific research documents, whether they are published or not. The documents may come from teaching and research institutions in France or abroad, or from public or private research centers.
L'archive ouverte pluridisciplinaire HAL, est destinée au dépôt et à la diffusion de documents scientifiques de niveau recherche, publiés ou non, émanant des établissements d'enseignement et de recherche français ou étrangers, des laboratoires publics ou privés. 


\title{
ASYMPTOTIC DETECTION PERFORMANCE OF THE ROBUST ANMF
}

\author{
Frédéric Pascal ${ }^{1}$ and Jean-Philippe Ovarlez $z^{2,3}$ \\ 1 L2S/CentraleSupélec-CNRS-Université Paris-Sud, 3 rue Joliot-Curie, 91190 Gif-sur-Yvette, France \\ 2 SONDRA/CentraleSupélec, Plateau du Moulon, 91190 Gif-sur-Yvette, France \\ 3 ONERA, DEMR/TSI, Chemin de la Hunière, 91120 Palaiseau, France
}

\begin{abstract}
This paper presents two different approaches to derive the asymptotic distributions of the robust Adaptive Normalized Matched Filter (ANMF) under both $H_{0}$ and $H_{1}$ hypotheses. More precisely, the ANMF has originally been derived under the assumption of partially homogenous Gaussian noise, i.e. where the variance is different between the observation under test and the set of secondary data. We propose in this work to relax the Gaussian hypothesis: we analyze the ANMF built with robust estimators, namely the $M$-estimators and the Tyler's estimator, under the Complex Elliptically Symmetric (CES) distributions framework. In this context, we derive two asymptotic distributions for this robust ANMF. Firstly, we combine the asymptotic properties of the robust estimators and the Gaussianbased distribution of the ANMF at finite distance. Secondly, we directly derive the asymptotic distribution of the robust ANMF.
\end{abstract}

Index Terms - Adaptive Normalized Match Filter, $M$-estimators, Tyler's estimator, Complex Elliptically Symmetric distributions, non-Gaussian detection, robust estimation theory.

\section{INTRODUCTION}

In the general statistical signal processing area, the detection problem is an important topic of research. For instance, one can cite the works in radar processing [1-4]. Since in practice, the noise parameters are unknown, an estimation step is required leading to the so-called adaptive detection processes. Among these unknown parameters, the noise Covariance Matrix (CM) is probably one of the most important since the resulting performance of adaptive detectors mainly relies on the estimation accuracy of this CM. This is the case for the Adaptive Matched Filter (AMF) [5], the Kelly's test [6] and the Adaptive Normalized Matched Filter (ANMF) [1]. Generally, the CM is estimated thanks to the so-called Sample Covariance Matrix (SCM). Although this estimator is very simple and provides optimal performance under a Gaussian noise, the resulting adaptive detector performance can strongly be degraded when the noise turned to be non-Gaussian, heterogeneous or when it contains outliers/jammers.

To fill these gaps, a general framework on robust estimation theory has been extensively studied in the statistical community in the 1970s following the seminal works of Huber and Maronna [7,8]. The multivariate real case has been recently extended to the complex case [9-11], more adapted for signal processing applications. Under this robust theory framework, most of recent works in CM estimation considers the broader class of Complex Elliptically Symmetric (CES) distributions. A complete review on CES applied to array processing can be found in [9].
In this CES framework, the so-called $M$-estimators [8] and the Tyler's estimator $[11,12]$ present alternatives to the Gaussian-based SCM. Although these robust estimators provide good results in practice [10], the statistical analysis of the resulting adaptive detectors is a difficult point. This is mainly due to the non explicit form of these estimators, defined through fixed point equations. However, their asymptotic properties have been recently derived in $[9,10]$. Following these works, the aim of this paper is to derive the asymptotic properties of the ANMF built with these estimators, namely the $M$-estimators and the Tyler's estimator, under both $H_{0}$ (absence of target) and $H_{1}$ (presence of target) hypotheses. The interest of such an analysis is to provide a better statistical characterization of the ANMF than the one based on the NMF [13].

The paper is organized as follows: next section provides the general background of this work as well as a recall on the main results concerning the statistics of the NMF and ANMF detection tests under both Gaussian assumption and CES distributions background. Section III gives without proof, the results presented in [14] concerning the asymptotic distribution of the ANMF built with robust estimators. Then, Section IV presents two different ways to derive the statistic of the ANMF built with any M-estimators for both $H_{0}$ and $H_{1}$ hypotheses. Finally, some conclusions and perspectives are drawn in the last section.

The following convention is adopted: italic indicates a scalar quantity, lower (resp. upper) case boldface indicates a vector (resp. matrix) quantity and upper case boldface a matrix. ${ }^{T}$ and ${ }^{H}$ represent respectively the transpose and the transpose conjugate operators, $\operatorname{Tr}($.$) denotes the trace operator, \mathbb{1}_{[0,1]}($.$) is the [0,1]$-indicator function, vec the vec operator and $\mathcal{C N}(\operatorname{resp} \mathcal{N})$ stands for the complex (resp. real) Gaussian distribution while $\mathcal{C E S}$ stands for the Complex Elliptically Symmetric distribution.

\section{BACKGROUND}

\subsection{The Normalized Matched Filter (NMF)}

Detecting a complex signal corrupted by an additive Gaussian noise $\mathbf{c} \sim \mathcal{C N}\left(\mathbf{0}, \sigma^{2} \mathbf{M}\right)$ in a $m$-dimensional complex observation vector $\mathbf{y}$ can be stated as the following binary hypothesis test:

$$
\left\{\begin{array}{lll}
H_{0}: \mathbf{y}=\mathbf{c} & \mathbf{y}_{i}=\mathbf{c}_{i} \quad i=1, \ldots, N \\
H_{1}: \mathbf{y}=\alpha \mathbf{p}+\mathbf{c} & \mathbf{y}_{i}=\mathbf{c}_{i} \quad i=1, \ldots, N
\end{array},\right.
$$

where $\mathbf{p}$ is a perfectly known complex steering vector, $\alpha$ is the unknown signal amplitude and where the $\mathbf{c}_{i} \sim \mathcal{C N}(\mathbf{0}, \mathbf{M})$ are $N$ signal-free independent measurements, traditionally called the secondary data, used to estimate the background CM M. When the CM 
$\mathbf{M}$ is known and the variance $\sigma^{2}$ is unknown, this binary hypothesis test is solved by the Generalized Likelihood Ratio Test (GLRT) theory leading to a well-known Normalized Matched Filter [13] denoted $H($.$) and defined on [0,1]$ by

$$
H(\mathbf{M})=\frac{\left|\mathbf{p}^{H} \mathbf{M}^{-1} \mathbf{y}\right|^{2}}{\left(\mathbf{p}^{H} \mathbf{M}^{-1} \mathbf{p}\right)\left(\mathbf{y}^{H} \mathbf{M}^{-1} \mathbf{y}\right)} .
$$

In order to derive the probability density function (PDF) of $H(\mathbf{M})$ under $H_{1}$ and $H_{0}$ hypothesis, let us set the signal-to-noise ratio (SNR) equal to $\delta=\alpha^{2} \mathbf{p}^{H} \mathbf{M}^{-1} \mathbf{p} / \sigma^{2}$. It can be shown in [4] that $H(\mathbf{M})$, derived for heterogeneous Gaussian environment, can be rewritten as $F /(F+1)$ where $(m-1) F$ is distributed according to a non-central $F$-distribution denoted by $F_{\alpha_{1}, \alpha_{2}, \delta}$ where $\alpha_{1}=1$, $\alpha_{2}=m-1$ and where $\delta$ is the noncentrality parameter. The PDF of $F$ is given by:

$$
p_{F}(u)=(m-1) e^{-\delta}(1+u)^{-m}{ }_{1} F_{1}\left(m, 1 ; \frac{u \delta}{u+1}\right),
$$

where ${ }_{1} F_{1}\left(\alpha_{1}, \alpha_{2} ;.\right)$ is the complex confluent hypergeometric function. After a simple change of variable, the distribution of $H(\mathbf{M})$ takes the following form:

$$
p_{H(\mathbf{M})}(u)=e^{-\delta} \beta_{1, m-1}(u)_{1} F_{1}(m, 1 ; u \delta),
$$

where $\beta_{1, m-1}(u)=(m-1)(1-u)^{m-2} \mathbb{1}_{[0,1]}(u)$ is the PDF of $\beta$ distribution with degrees of freedom 1 and $m-1$, denoted $\beta(1, m-$ $1)$. Thus, setting $\delta$ to zero simply leads to the $\beta(1, m-1)$ PDF for $H(\mathbf{M})$ under $H_{0}$ hypothesis. Hence, the theoretical relationship between the detection threshold $\lambda$ and the Probability of False Alarm (PFA) is defined as:

$$
P_{f a}=\mathbb{P}\left(H(\mathbf{M})>\lambda \mid H_{0}\right)=(1-\lambda)^{m-1} .
$$

This relation will serve as a benchmark since it characterizes the case of a perfectly known covariance matrix for the detection test. When $\delta \neq 0$, the Probability of Detection $P_{d}$ for a given SNR $\delta$ and for a fixed value of the detection threshold $\lambda$ is given by:

$$
\begin{aligned}
P_{d} & =\mathbb{P}\left(H(\mathbf{M})>\lambda \mid H_{1}\right) \\
& =1-e^{-\delta} \int_{0}^{\lambda} \beta_{1, m-1}(u)_{1} F_{1}(m, 1 ; u \delta) d u .
\end{aligned}
$$

Note that, for efficient numerical computation of the previous equation (for any non-zero noncentrality parameter of the confluent hypergeometric function), it is better to use the following relationship:

$$
{ }_{1} F_{1}(a, b ; z)=e^{z}{ }_{1} F_{1}(b-a, b ;-z),
$$

leading to

$$
P_{d}=1-\int_{0}^{\lambda} \beta_{1, m-1}(u) e^{\delta(u-1)}{ }_{1} F_{1}(1-m, 1 ;-u \delta) d u .
$$

\subsection{The Adaptive Normalized Matched Filter (ANMF)}

When an estimate $\widehat{\mathbf{M}}$ of the CM M is plugged into the NMF (twostep GLRT), this results in the so-called ANMF or ACE (Adaptive Coherence Estimator) [1,3]. Assuming that the SCM, defined as $\widehat{\mathbf{M}}_{S C M}=\frac{1}{N} \sum_{k=1}^{N} \mathbf{c}_{k} \mathbf{c}_{k}^{H}$ is used, Kraut et al. have shown in [4] that $H\left(\widehat{\mathbf{M}}_{S C M}\right)$ has the same distribution as $\frac{\hat{F}}{\hat{F}+1}$ where $\hat{F} \sim$
$\frac{\mathcal{C} \chi_{1}^{2}(\delta)}{\mathcal{C} \chi_{N-m+1}^{2}(0)} \frac{1}{1-b}$ and where $b \sim \beta(N-m+2, m-1)$. After simple but fastidious derivations, one obtains:

$$
\begin{aligned}
f_{H\left(\widehat{\mathbf{M}}_{S C M}\right)}(x) & =\frac{e^{-\delta}}{K} \int_{0}^{1} u^{N-m+1} \frac{(1-u)^{m-1}(1-x)^{N-m}}{(1-u x)^{N-m+2}} \\
\times{ }_{1} F_{1} & \left(N-m+2,1 ; \frac{\delta x(1-u)}{1-x u}\right) d u
\end{aligned}
$$

where $K=\frac{\Gamma(N-m+1) \Gamma(m-1)}{\Gamma(N+1)}$. By setting $\delta=0$ in the previous equation, the resulting PDF $f_{H\left(\widehat{\mathbf{M}}_{S C M}\right)}$ of $H\left(\widehat{\mathbf{M}}_{S C M}\right)$ under $H_{0}$ hypothesis can be retrieved [15]:

$$
\begin{gathered}
f_{H\left(\widehat{\mathbf{M}}_{S C M}\right)}(x)=\frac{(N-m+1)(m-1)}{N+1}(1-x)^{N-m} \\
\quad \times{ }_{2} F_{1}(N-m+2 ; N-m+2 ; N+2 ; x),
\end{gathered}
$$

where ${ }_{2} F_{1}($.$) is the hypergeometric function [16]. The theoretical$ relationship between the detection threshold $\lambda$ and the Probability of False Alarm $P_{f a}$ is defined as:

$$
\begin{aligned}
P_{f a} & =\mathbb{P}\left(H\left(\widehat{\mathbf{M}}_{S C M}\right)>\lambda \mid H_{0}\right) \\
& =(1-\lambda)^{N-m+1}{ }_{2} F_{1}(N-m+2, N-m+1 ; N+1 ; \lambda),
\end{aligned}
$$

whereas the corresponding relationship between $P_{d}$ and the SNR $\delta$ for a fixed $P_{f a}$ leads to:

$$
\begin{aligned}
P_{d}=\mathbb{P} & \left(H\left(\widehat{\mathbf{M}}_{S C M}\right)>\lambda \mid H_{1}\right) \\
=1 & -\frac{e^{-\delta}}{K} \int_{0}^{1} d u \int_{0}^{\lambda} u^{N-m+1} \frac{(1-u)^{m-1}(1-x)^{N-m}}{(1-u x)^{N-m+2}} \\
& \times{ }_{1} F_{1}\left(N-m+2,1 ; \frac{\delta x(1-u)}{1-x u}\right) d x .
\end{aligned}
$$

\subsection{M-estimators, Tyler's estimator and asymptotic properties}

This section presents the $M$-estimators, the Tyler's estimator as well as their asymptotic properties. Details of the following results can be found in $[9,10]$ for $M$-estimators and in $[11,12,17]$ for the Tyler's estimator.

In the literature of radar detection and estimation, Spherically Invariant Random Vector (SIRV) modeling and Complex Elliptical Symmetric distributions (CES), originally introduced by Kelker in [18], have been considered and have been studied for their good statistical properties and for their good fitting to experimental nonGaussian radar data [19]. They provide a multivariate location-scale family of distributions that primarily serve as long tailed alternatives to the multivariate Gaussian model. A good review on these distributions can be found in $[9,20]$. Let $\mathbf{c}$ be a $m$-dimensional complex random vector. $\mathbf{c}$ follows a CES distribution if its PDF can be written as

$$
g_{\mathbf{c}}(\mathbf{c})=\left|\boldsymbol{\Sigma}^{-1}\right| h_{\mathbf{c}}\left((\mathbf{c}-\boldsymbol{\mu})^{H} \boldsymbol{\Sigma}^{-1}(\mathbf{c}-\boldsymbol{\mu})\right),
$$

where $h_{\mathbf{c}}: \mathbb{R}^{+} \rightarrow \mathbb{R}^{+}$is any function such that (13) defines a PDF, $\boldsymbol{\mu}$ is the statistical mean and $\boldsymbol{\Sigma}$ is a scatter matrix. It will be denoted $\mathbf{c} \sim \mathcal{C} \mathcal{E S}(\boldsymbol{\mu}, \boldsymbol{\Sigma}) . \boldsymbol{\Sigma}$ reflects the structure of the $\mathrm{CM}$ of $\mathbf{c}$, i.e. the covariance matrix is equal to $\boldsymbol{\Sigma}$ up to a scale factor. One can notice that the Gaussian distribution is a particular case of CES. In this paper, we will assume that $\boldsymbol{\mu}=\mathbf{0}$ and without loss of generality, the scatter matrix will be taken equal to the CM M. 
Now, let $\left(\mathbf{c}_{1}, \ldots, \mathbf{c}_{N}\right)$ be a $N$-sample of $m$-dimensional complex independent vectors with $\mathbf{c}_{k} \sim \mathcal{C} \mathcal{E S}(\mathbf{0}, \mathbf{M}), k=1, \ldots, N$. The $M$-estimators are defined as the unique solution of the following equation

$$
\widehat{\mathbf{M}}=\frac{1}{N} \sum_{k=1}^{N} u\left(\mathbf{c}_{k}^{H} \widehat{\mathbf{M}}^{-1} \mathbf{c}_{k}\right) \mathbf{c}_{k} \mathbf{c}_{k}^{H},
$$

where $u($.$) stands for any real-valued function that satisfies a set$ of general assumptions (see $[9,10]$ ), mainly for ensuring the existence, uniqueness and convergence of the previous equation. Note that MLEs are particular solutions of the previous equation.

An attractive and powerful estimator, independent of the CES distribution, is the Tyler's estimator also called the Fixed Point and defined as the solution of

$$
\widehat{\mathbf{M}}=\frac{m}{N} \sum_{k=1}^{N} \frac{\mathbf{c}_{k} \mathbf{c}_{k}^{H}}{\mathbf{c}_{k}^{H} \widehat{\mathbf{M}}^{-1} \mathbf{c}_{k}} .
$$

For all $M$-estimator $\widehat{\mathbf{M}}$ which verifies equation (14), one has the important asymptotical statistical behavior:

$$
\sqrt{N}(\operatorname{vec}(\widehat{\mathbf{M}}-\mathbf{M})) \stackrel{d}{\longrightarrow} \mathcal{G C N}\left(\mathbf{0}_{m^{2}, 1}, \boldsymbol{\Sigma}_{M}, \boldsymbol{\Omega}_{M}\right),
$$

where $\mathbf{M}$ is the consistent limit of $\widehat{\mathbf{M}}$ and $\mathcal{G C N}\left(\mathbf{0}, \boldsymbol{\Sigma}_{M}, \boldsymbol{\Omega}_{M}\right)$ denotes the Generalized Complex Normal distribution with $\boldsymbol{\Sigma}_{M}$ the covariance matrix and $\boldsymbol{\Omega}_{M}$ the pseudo-covariance matrix defined as

$$
\begin{aligned}
& \boldsymbol{\Sigma}_{M}=\nu_{1} \mathbf{M}^{T} \otimes \mathbf{M}+\nu_{2} \operatorname{vec}(\mathbf{M}) \operatorname{vec}(\mathbf{M})^{H}, \\
& \boldsymbol{\Omega}_{M}=\nu_{1}\left(\mathbf{M}^{T} \otimes \mathbf{M}\right) \mathbf{K}+\nu_{2} \operatorname{vec}(\mathbf{M}) \operatorname{vec}(\mathbf{M})^{T},
\end{aligned}
$$

where $\mathbf{K}$ is the commutation matrix which transforms $\operatorname{vec}(\mathbf{A})$ into $\operatorname{vec}\left(\mathbf{A}^{T}\right), \nu_{1}$ and $\nu_{2}$ are real scalars relying on the CES distribution and given in $[9,21]$.

It is important to notice that the previous result is also valid for the SCM when the observations are Gaussian $\left(\nu_{1}=1\right.$ and $\nu_{2}=0$, see e.g. [22]) and for the Tyler's estimator for CES-distributed observations $\left(\nu_{1}=(m+1) / m\right.$ and $\nu_{2}=-(m+1) / m^{2}$, see e.g. [17]). This shows that the asymptotic behavior of all these estimators is similar. More precisely, the $M$-estimators and the Tyler's estimator behaves asymptotically the same as the SCM, it differs only from the scalar quantities $\nu_{1}$ and $\nu_{2}$.

\subsection{Asymptotic properties of the ANMF built with M-estimates}

The asymptotic behavior of all the presented estimators can then be extended to the ANMF thanks to the following result.

Let $H($.$) be a r$-dimensional multivariate function on the set of $m \times m$ positive-definite symmetric matrices with continuous first partial derivatives and such as $H(\mathbf{M})=H(\alpha \mathbf{M})$ for all $\alpha>0$, i.e. $H($.$) is homogeneous of degree 0$. For all $\widehat{\mathbf{M}}$ that verifies equation (16), one has the following result, derived in $[9,21]$ :

$$
\sqrt{N}(H(\widehat{\mathbf{M}})-H(\mathbf{M})) \stackrel{d}{\longrightarrow} \mathcal{G C N}\left(\mathbf{0}_{r, 1}, \boldsymbol{\Sigma}_{H}, \boldsymbol{\Omega}_{H}\right),
$$

where $\boldsymbol{\Sigma}_{H}$ and $\boldsymbol{\Omega}_{H}$ are defined as

$$
\begin{aligned}
& \boldsymbol{\Sigma}_{H}=\nu_{1} H^{\prime}(\mathbf{M})\left(\mathbf{M}^{T} \otimes \mathbf{M}\right) H^{\prime}(\mathbf{M})^{H}, \\
& \boldsymbol{\Omega}_{H}=\nu_{1} H^{\prime}(\mathbf{M})\left(\mathbf{M}^{T} \otimes \mathbf{M}\right) \mathbf{K} H^{\prime}(\mathbf{M})^{T},
\end{aligned}
$$

and $H^{\prime}(\mathbf{M})=\frac{\partial H(\mathbf{M})}{\partial \operatorname{vec}(\mathbf{M})}=\left(h_{i j}^{\prime}\right)$ with $h_{i j}^{\prime}=\frac{\partial h_{i}}{\partial m_{j}}$ and $m_{j}$ 's denote the elements of $\operatorname{vec}(\mathbf{M})$, for $j=1, \ldots, m^{2}$.

When comparing to the asymptotic behavior of any function $H$ with SCM argument $\mathbf{M}$, one obtains $\nu_{1}=1$. For any function $H$ with Tyler's argument $\mathbf{M}$, we obtain $\nu_{1}=(m+1) / m$. This explains that any function $H$ of $M$-estimators has the same asymptotic distribution than those of a Wishart matrix (SCM) with $N / \nu_{1}$ degrees of freedom. It could also be interpreted as follows: under Gaussian assumption, $M$-estimators require $\nu_{1} N$ secondary data to reach the same performance as the SCM.

\section{ASYMPTOTIC BEHAVIOR OF THE ANMF TEST}

The goal of this section is to propose two different ways of deriving an approximate distribution of the test $H(\widehat{\mathbf{M}})$ built with any $M$ estimators under both $H_{0}$ and $H_{1}$ hypotheses. The first approach consists in using the asymptotic distribution presented in section 2.4 for the different estimators while the second approach is to compute analytically the parameters $\boldsymbol{\Sigma}_{H}$ and $\boldsymbol{\Omega}_{H}$ characterizing the asymptotic distribution of the ANMF given by equation (18).

\subsection{Correction of the degrees of freedom, compared to the Gaussian-based SCM}

Let us first consider the two ANMF PDF given by (9) under $H_{1}$ hypothesis and (10) under $H_{0}$ hypothesis. Note that these two equations provide the exact distributions of $H\left(\widehat{\mathbf{M}}_{S C M}\right)$ under both $H_{0}$ and $H_{1}$ hypotheses when the observations $\mathbf{y}, \mathbf{y}_{1}, \ldots, \mathbf{y}_{N}$ are Gaussian distributed. Now, for $N$ sufficiently large, equation (16) states that a $M$-estimator built with $N \nu_{1}$ observations behaves as the SCM built with $N$ observations. Consequently, combining this result with equations (9) and (10) or equivalently with equations (11) and (12), leads to the approximate distribution for $H(\widehat{\mathbf{M}})$ under both hypotheses where $\widehat{\mathbf{M}}$ stands for any $M$-estimator or for the Tyler's estimator. The final theoretical relationship between the detection threshold $\lambda$ and $P_{f a}=\mathbb{P}\left(H(\widehat{\mathbf{M}})>\lambda \mid H_{0}\right)$ and the theoretical relationship between $\lambda$ and $P_{d}=\mathbb{P}\left(H(\widehat{\mathbf{M}})>\lambda \mid H_{1}\right)$ are therefore given by

$$
P_{f a}=(1-\lambda)^{a-1}{ }_{2} F_{1}(a, a-1 ; b-1 ; \lambda),
$$

and

$$
\begin{aligned}
P_{d}=1 & -\frac{e^{-\delta}}{K} \int_{0}^{1} d u \int_{0}^{\lambda} u^{a-1} \frac{(1-u)^{m-1}(1-x)^{a-2}}{(1-u x)^{a}} \\
& \times{ }_{1} F_{1}\left(a, 1 ; \frac{\delta x(1-u)}{1-x u}\right) d x
\end{aligned}
$$

where $K=\frac{\Gamma(a-1) \Gamma(m-1)}{\Gamma(b-1)}, a=N / \nu_{1}-m+2$ and $b=$ $N / \nu_{1}+2$.

As illustrated in the simulations and although no rigorous proof is given, the previous result provides a very accurate PDF for $H(\widehat{\mathbf{M}})$ even for small $N$.

\subsection{Asymptotic covariance of the ANMF}

Let us now turn to the asymptotic distribution of the ANMF for any $\mathrm{CM}$ estimator.

Proposition 3.1 Let us consider the ANMF test defined by

$$
H(\widehat{\mathbf{M}})=\frac{\left|\mathbf{p}^{H} \widehat{\mathbf{M}}^{-1} \mathbf{y}\right|^{2}}{\left(\mathbf{p}^{H} \widehat{\mathbf{M}}^{-1} \mathbf{p}^{H}\right)\left(\mathbf{y}^{H} \widehat{\mathbf{M}}^{-1} \mathbf{y}\right)} .
$$

This function $H($.$) is homogeneous of degree 0$ and one can apply result given in (18). For any estimator $\widehat{\mathbf{M}}$ satisfying equation 
(16), one has $\sqrt{N}(H(\widehat{\mathbf{M}})-H(\mathbf{M})) \stackrel{d}{\longrightarrow} \mathcal{N}\left(0, \boldsymbol{\Sigma}_{H}\right)$, where the asymptotic variance $\boldsymbol{\Sigma}_{H}$ and pseudo-covariance $\boldsymbol{\Omega}_{H}$ of the ANMF statistic is given by

$$
\boldsymbol{\Sigma}_{H}=\boldsymbol{\Omega}_{H}=2 \nu_{1} H(\mathbf{M})(H(\mathbf{M})-1)^{2} .
$$

Proof 3.1 The proof has been recently derived in [14] and is omitted here.

Notice that, contrary to the first approach, the previous asymptotic distribution is a distribution conditional to the observation $\mathbf{y}$ that appears in $H(\mathbf{M})$. Consequently, a supplementary step is required to obtain the asymptotic distribution of $H(\widehat{\mathbf{M}})$. Let us rewrite the result of Proposition 3.1 as

$$
H(\widehat{\mathbf{M}}) \stackrel{d}{\longrightarrow} \mathcal{N}\left(H(\mathbf{M}), \frac{2 \nu_{1}}{N} H(\mathbf{M})(H(\mathbf{M})-1)^{2}\right)
$$

\section{EVALUATION OF PERFORMANCES}

\subsection{Evaluation of asymptotic performances under $H_{0}$}

According to result given by (24), for $N$ large enough, considering that $H(\widehat{\mathbf{M}}) \sim \mathcal{N}\left(X, \sigma_{X}^{2}\right)$ where $\sigma_{X}^{2}=\frac{2 \nu_{1}}{N} X(X-1)^{2}$ and $X=$ $H(\mathbf{M}) \sim \beta(1, m-1)$, one can obtain the asymptotic distribution $f_{H(\widehat{\mathbf{M}})}^{a}$ of $H(\widehat{\mathbf{M}})$ as follows

$$
f_{H(\widehat{\mathbf{M}})}^{a}(u)=\int_{0}^{1} \frac{\sqrt{N} \exp \left(-\frac{N(u-x)^{2}}{4 \nu_{1} x(x-1)^{2}}\right)}{\sqrt{4 \pi \nu_{1} x(x-1)^{2}}} \beta_{1, m-1}(x) d x .
$$

Now, if we denote $\Phi($.$) the cumulative distribution of the Nor-$ mal distribution, one obtains the corresponding asymptotical $P_{f a}-\lambda$ relationship:

$$
P_{f a}=1-\int_{0}^{1} \beta_{1, m-1}(x) \Phi\left(\frac{\sqrt{N}(\lambda-x)}{\sqrt{2 \nu_{1} x(x-1)^{2}}}\right) d x
$$

\subsection{Evaluation of asymptotic performances under $H_{1}$}

Here, we assume that the noise in the cell under test is Gaussian distributed whereas the secondary data are CES distributed. In that case, due to the fact that the $M$-estimator is independent of the CES distribution, the distribution of $H(\widehat{\mathbf{M}})$ under $H_{1}$ hypothesis is given by $p_{H(\mathbf{M})}$ in equation (4). According to the result given by (24), for $N$ large enough, considering that $H(\widehat{\mathbf{M}}) \sim \mathcal{N}\left(X, \sigma_{X}^{2}\right)$ where $\sigma_{X}^{2}=\frac{2 \nu_{1}}{N} X(X-1)^{2}$ and $X=H(\mathbf{M}) \sim p_{H(\mathbf{M})}$, one can obtain the asymptotic distribution $f_{H(\widehat{\mathbf{M}})}^{a}$ of $H(\widehat{\mathbf{M}})$ as follows

$$
f_{H(\widehat{\mathbf{M}})}^{a}(u)=\int_{0}^{1} \frac{\sqrt{N} \exp \left(-\frac{N(u-x)^{2}}{4 \nu_{1} x(x-1)^{2}}\right)}{\sqrt{4 \pi \nu_{1} x(x-1)^{2}}} p_{H(\mathbf{M})}(x) d x .
$$

where $p_{H(\mathbf{M})}($.$) is given by (4). Following the same reasoning than$ in the previous subsection, one obtains, for a given detection threshold $\lambda$, the corresponding asymptotical $P_{d^{-}} \delta$ relationship:

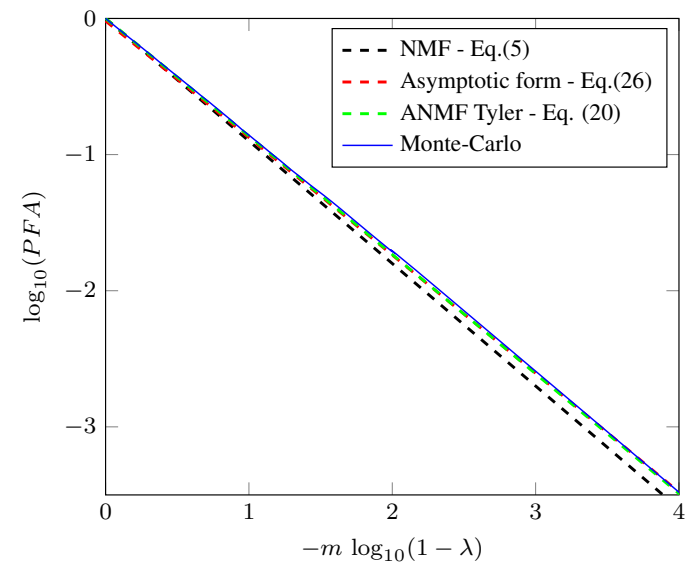

Fig. 1. Comparison between PFA-threshold relationships for the NMF, the ANMF built with the Tyler's estimator and its asymptotic form, $m=10, N=200, \nu_{1}=1.1, \mathbf{p}=[1, \ldots, 1]^{T}$, $\mathbf{y} \sim K_{\nu}$ where $K_{\nu}$ is a multivariate K-distribution with shape parameter $\nu=0.1$ and covariance matrix $\mathbf{M}$.

$$
\begin{aligned}
P_{d}=1 & -\int_{0}^{1} \beta_{1, m-1}(x) e^{\delta(x-1)}{ }_{1} F_{1}(1-m, 1 ;-x \delta) \\
& \times \Phi\left(\frac{\sqrt{N}(\lambda-x)}{\sqrt{2 \nu_{1} x(x-1)^{2}}}\right) d x
\end{aligned}
$$

In the case where the cell under test contains CES noise, the PDF $p_{H(\mathbf{M})}$ of $H(\widehat{\mathbf{M}})$ under $H_{1}$ hypothesis is no more given by (4) and the performance of the test becomes very complicated to derive.

\section{SIMULATIONS}

In this section, we set the Toeplitz CM M whose entries are defined as $M_{i j}=\rho^{|i-j|}$ where $\rho$ is equal to 0.5 . Figure 1 shows the PFAthreshold relationships for the NMF given by (5), the first approximate distribution of the ANMF built with Tyler's estimator given by (20) and the asymptotic expression derived in (26) for the Tyler's estimator and the empirical PFA for the Tyler-ANMF, for $N=200$ and $m=10$, for K-distributed secondary data with shape parameter $\nu=0.1$. First, the asymptotic regime is achieved and one can observe a good agreement between the two asymptotic distributions derived in this paper. Moreover, this shows that these two approximations provide a very good characterization of the Tyler-ANMF behavior (solid blue line).

Figure 2 shows the $P_{d}$-SNR relationships for the NMF (Equation (6) or (8)), the first approximate distribution of the ANMF built with Tyler's estimator (21), the asymptotic expression derived in (28) for the Tyler's estimator and the empirical $P_{d}$ for the Tyler-ANMF for $m=10, N=500$, for a PFA equal to $P_{f a}=10^{-3}$, for Kdistributed secondary data with shape parameter $\nu=0.1$ and for a cell under test containing Gaussian noise. The asymptotic regime is achieved and one can observe a good agreement between the two asymptotic distributions derived in this paper.

\section{CONCLUSION}

In the context of robust detection in Gaussian or non-Gaussian noise, two asymptotic distributions of the ANMF have been proposed for 


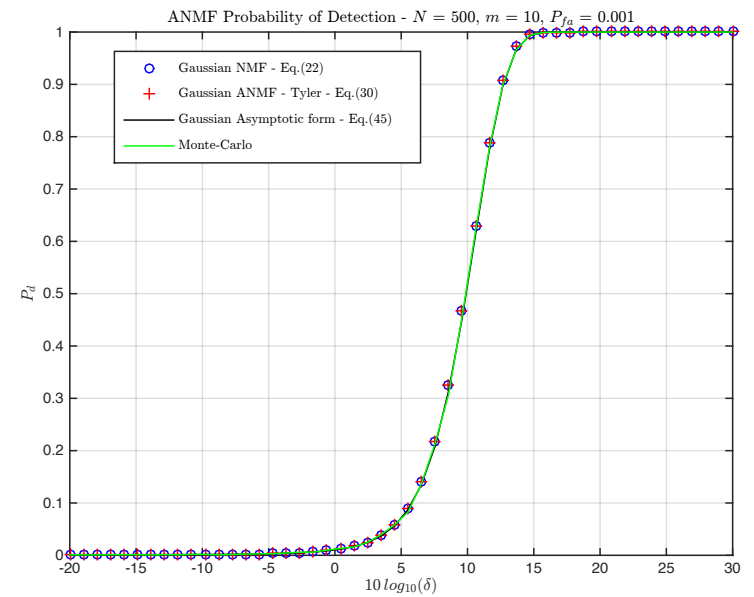

Fig. 2. Comparison between $P_{d}$ and SNR $\delta$ relationships for the ANMF built with Tyler's estimator, $m=10, N=500$ and $P_{f a}=10^{-3}, \mathbf{p}=[1, \ldots, 1]^{T},\left\{\mathbf{y}_{i}\right\}_{i \in[1, N]} \sim K_{\nu}$ where $K_{\nu}$ is a K-distribution with shape $\nu=0.1$. and $\mathbf{y} \sim \mathcal{C N}(\alpha \mathbf{p}, \mathbf{M})$

both $H_{0}$ and $H_{1}$ hypotheses. More precisely, using robust CM estimators such as $M$-estimators or the Tyler's estimator, two asymptotic approximations of the corresponding ANMF distribution have been derived following different approaches. First, we have combined the exact distribution of the ANMF built with the SCM under Gaussian noise and the asymptotic properties of the robust estimators. Finally, we have directly derived the asymptotic distribution of the robust ANMF under CES environment. These results provide a very good approximation of the ANMF distribution even for a small number of observations and have been applied to theoretically regulate the false alarm probability and to evaluate the detection performance. However, all these results are obtained considering a cell under test containing Gaussian noise and secondary data characterized by CES noise. In the case where the cell under test is no more Gaussian, further works have to be conducted.

\section{REFERENCES}

[1] E. Conte and M. Lops and G. Ricci, "Asymptotically Optimum Radar Detection in Compound-Gaussian Clutter," IEEE Transactions on Aerospace and Electronic Systems, vol. 31, no. 2, pp. 617-625, 1995.

[2] F. Gini, "Sub-Optimum Coherent Radar Detection in a Mixture of K-Distributed and Gaussian Clutter," IEE Proc. on Radar, Sonar Navig., vol. 144, pp. 39-48, February 1997.

[3] S. Kraut and L. L. Scharf, "The CFAR adaptive subspace detector is a scale-invariant GLRT," Signal Processing, IEEE Transactions on, vol. 47, no. 9, pp. 2538-2541, 1999.

[4] S. Kraut, L. Scharf, and L. M. Whorter, "Adaptive Subspace Detector," Signal Processing, IEEE Transactions on, vol. 49, pp. 1-16, January 2001.

[5] F. C. Robey, D. R. Fuhrmann, E. J. Kelly, and R. Nitzberg, "A CFAR adaptive matched filter detector," Aerospace and Electronic Systems, IEEE Transactions on, vol. 28, no. 1, pp. 208216, January 1992.
[6] E. J. Kelly, "An adaptive detection algorithm," Aerospace and Electronic Systems, IEEE Transactions on, no. 2, pp. 115-127, 1986.

[7] P. J. Huber, "Robust estimation of a location parameter," The Annals of Mathematical Statistics, vol. 35, no. 1, pp. 73-101, 1964.

[8] R. A. Maronna, "Robust $M$-estimators of multivariate location and scatter," Annals of Statistics, vol. 4, no. 1, pp. 51-67, January 1976.

[9] E. Ollila, D. E. Tyler, V. Koivunen, and H. V. Poor, "Complex elliptically symmetric distributions: Survey, new results and applications," Signal Processing, IEEE Transactions on, vol. 60, no. 11, pp. $5597-5625$, nov. 2012.

[10] M. Mahot, F. Pascal, P. Forster, and J.-P. Ovarlez, "Asymptotic properties of robust complex covariance matrix estimates," Signal Processing, IEEE Transactions on, vol. 61, no. 13, pp. 3348-3356, July 2013.

[11] F. Pascal, Y. Chitour, J.-P. Ovarlez, P. Forster, and P. Larzabal, "Covariance structure maximum-likelihood estimates in compound Gaussian noise: existence and algorithm analysis," Signal Processing, IEEE Transactions on, vol. 56, no. 1, pp. 34-48, Jan. 2008.

[12] D. Tyler, "A distribution-free $m$-estimator of multivariate scatter," The Annals of Statistics, vol. 15, no. 1, pp. 234-251, 1987.

[13] L. L. Scharf and D. W. Lytle, "Signal detection in Gaussian noise of unknown level: an invariance application," Information Theory, IEEE Transactions on, vol. 17, pp. 404-411, July 1971.

[14] F. Pascal and J.-P. Ovarlez, "Asymptotic Properties of the Robust ANMF," in IEEE International Conference on Acoustics, Speech, and Signal Processing, ICASSP-15, Brisbane, Australia, April 2015.

[15] F. Pascal, J.-P. Ovarlez, P. Forster, and P. Larzabal, "Constant false alarm rate detection in spherically invariant random processes," in Proc. of the European Signal Processing Conf., Vienna, September 2004, pp. 2143-2146.

[16] M. Abramowitz and I. Stegun, Handbook of Mathematical Functions. National Bureau of Standard, AMS 55, June 1964.

[17] F. Pascal, P. Forster, J.-P. Ovarlez, and P. Larzabal, "Performance analysis of covariance matrix estimates in impulsive noise," Signal Processing, IEEE Transactions on, vol. 56, no. 6, pp. 2206-2217, Jun. 2008.

[18] D. Kelker, "Distribution theory of spherical distributions and a location-scale parameter generalization," Sankhyā: The Indian Journal of Statistics, Series A, vol. 32, no. 4, pp. 419-430, 1970.

[19] E. Conte and A. De Maio, "Mitigation techniques for nonGaussian sea clutter," Oceanic Engineering, IEEE Journal of, vol. 29, no. 2, pp. 284-302, 2004.

[20] G. Frahm, "Generalized elliptical distributions: Theory and applications," Ph.D. dissertation, Universität zu Köln, 2004.

[21] M. Mahot, F. Pascal, P. Forster, and J.-P. Ovarlez, "Asymptotic properties of robust covariance matrix estimates," Signal Processing, IEEE Transactions on, vol. 61, no. 13, pp. 3348-3356, 2013.

[22] T. W. Anderson, An Introduction to Multivariate Statistical Analysis, 1st ed. John Wiley \& Sons, New York, 1958. 